\title{
Plasma ACE2 activity is persistently elevated following SARS-CoV-2 infection: implications for COVID-19 pathogenesis and consequences
}

\author{
To the Editor:
}

Coronavirus disease 2019 (COVID-19) causes persistent endothelial inflammation, lung, cardiovascular, kidney and neurological complications, and thromboembolic phenomena of unclear pathogenesis [1]. Severe acute respiratory syndrome-coronavirus-2 (SARS-CoV-2) utilises the catalytic site of full-length membrane-bound angiotensin converting enzyme 2 (ACE2) for host cell entry [2], which is thought to downregulate membrane-bound ACE2, and thus contribute to ongoing inflammation due to loss of a degradative pathway for angiotensin II. In healthy individuals, ACE2 exists primarily in its membrane-bound form with very low levels of the catalytically active ectodomain of ACE2 present in the circulation [3]. However, in patients with cardiovascular disease, there is increased "shedding" of ACE2, and higher circulating levels are associated with downregulation of membrane-bound ACE2 [4].

In this prospective observational study in recovered COVID-19 patients, we hypothesised that SARS-CoV-2 infection would be associated with shedding of ACE2 from cell membranes leading to increased plasma ACE2 activity levels.

We measured plasma ACE2 catalytic activity using a validated, sensitive quenched fluorescent substrate-based in-house assay as previously described [3-5] in a cohort of Australians aged $\geqslant 18$ years $(\mathrm{n}=66)$ who had recovered from mild, moderate or severe SARS-CoV-2 infection (positive result by PCR testing) and age- and gender-matched uninfected controls $(n=70)$. Of the 66 recovered SARS-CoV-2 patients, 23 agreed to provide follow-up samples. The severity of COVID-19 infection was defined according to National Institutes of Health COVID-19 treatment guidelines [6] as mild (predominantly upper respiratory tract infection), moderate (lower respiratory tract infection) or severe (hospitalisation). The immune responses in a subset of the overall cohort in this paper have been previously reported [7], and for the purpose of this study, we also obtained information on medications and comorbid conditions including hypertension (diagnosis of hypertension and/or anti-hypertensive treatment), hyperlipidaemia (treatment with lipid lowering agents), obesity (body mass index $\geqslant 30 \mathrm{~kg} \cdot \mathrm{m}^{-2}$ ), diabetes and cardiovascular disease. The study was approved by the University of Melbourne Human Research Ethics Committee (\#2056689); all participants provided written informed consent in accordance with the Declaration of Helsinki.

Data were analysed with SPSS version 26.0 (IBM SPSS Statistics, IBM Corp., USA). Plasma ACE2 activity was compared using the Mann-Whitney test or Kruskal-Wallis test and significance adjusted by Bonferroni correction for multiple tests. Fisher's exact test was used to compare categorical variables. Serial ACE2 activity levels were analysed using the Friedman test for repeated measures; after post hoc analysis with Wilcoxon signed-rank tests with a Bonferroni correction applied. Two-tailed p-values $<0.05$ were considered significant.

The controls and SARS-CoV-2 recovered subjects were matched for age (mean \pm SD $54 \pm 11$ versus $53 \pm 14$ years; $\mathrm{p}=0.47)$ and gender (37 (53\%) versus 39 (59\%) male; $\mathrm{p}=0.49)$. With regard to risk factors and/or comorbidities, hypertension was present in $7 \%(n=9)$ of all subjects, hyperlipidaemia in $8 \%(n=11)$, obesity

@ERSpublications

Plasma ACE2 activity is persistently elevated in patients after COVID-19 infection. Larger studies are needed to determine if this identifies people at risk of prolonged illness following COVID-19. https:// bit.ly/2XQlrYF

Cite this article as: Patel SK, Juno JA, Lee WS, et al. Plasma ACE2 activity is persistently elevated following SARS-CoV-2 infection: implications for COVID-19 pathogenesis and consequences. Eur Respir J 2021; 57: 2003730 [https://doi.org/10.1183/13993003.03730-2020]. 
in $2 \%(n=3)$, diabetes in $1 \%(n=1)$ and cardiovascular disease in $2 \%(n=3)$, with no difference between controls and SARS-CoV-2 recovered subjects (all, $\mathrm{p}>0.05$ ). There was no difference in the use of antihypertensive $(n=7)$, lipid lowering $(n=11)$ or antiplatelet therapy $(n=4)$ between controls and SARS-CoV-2 recovered subjects (all, $\mathrm{p}>0.05$ ).

At a median of 35 days post-infection (interquartile range 30-38 days), plasma ACE2 activity was significantly higher in recovered SARS-CoV-2 subjects compared to controls (5.8 (2-11.3) versus 0.06 $(0.02-2.2) \mathrm{pmol} \cdot \mathrm{min}^{-1} \cdot \mathrm{mL}^{-1} ; \mathrm{p}<0.0001$ ) (figure 1a). Plasma ACE2 activity differed according to disease severity ( $\mathrm{p}=0.033)$, with severe COVID-19 associated with higher ACE2 activity compared to mild disease (11.2 (8.3-23.2) versus $\left.5.4(1.8-9.0) \mathrm{pmol} \cdot \mathrm{min}^{-1} \cdot \mathrm{mL}^{-1} ; \mathrm{p}=0.027\right)$ (figure $1 \mathrm{~b}$ ). Men $(\mathrm{n}=39$ ) who were SARS-CoV-2-positive had higher median plasma ACE2 levels compared to women ( $\mathrm{n}=27)$ (9.2 (5.8-15.3) versus $\left.2.1(0.2-5.1) \mathrm{pmol} \cdot \mathrm{min}^{-1} \cdot \mathrm{mL}^{-1} ; \mathrm{p}<0.0001\right)$. The observation that plasma ACE2 is higher in men compared to women is consistent with our previous findings in non-COVID-19 patients with coronary artery disease [5], and in the general population [8].

We next analysed whether elevated plasma ACE2 activity persisted following SARS-CoV-2 infection in 23 subjects who provided further blood samples at 63 (56-65) and 114 (111-125) days post-infection. This subset was representative of the overall cohort for age (52 \pm 15 years), gender (14 (61\%) male) and disease severity (mild 15 (65\%), moderate 5 (22\%), severe 3 (13\%)). Plasma ACE2 activity remained persistently elevated in almost all subjects (figure 1c), with no significant differences between time-points in post hoc comparisons $(p>0.05)$. There was no change in plasma ACE2 activity in control subjects with serial samples ( $\mathrm{n}=8$; timepoint 1: $0.8(0.02-3.0)$; versus timepoint 2: $\left.1.4(0.02-5.9) \mathrm{pmol} \cdot \mathrm{min}^{-1} \cdot \mathrm{mL}^{-1} ; \mathrm{p}=0.31\right)$.

This is the first description that plasma ACE2 activity is elevated in patients after COVID-19 infection compared to uninfected age- and gender-matched controls with similar comorbidities, and the first with long-term data to indicate that plasma ACE2 activity remains elevated out to a median of 114 days post-infection, the last time-point sampled. It is already known that males have higher plasma ACE2 activity than females $[5,8]$, but this is the first report that plasma ACE2 activity levels are increased in those with more severe COVID-19 infection. Although the pathophysiological causes and consequences of elevated plasma ACE2 activity in the setting of COVID-19 are unknown, we have reported that increased plasma ACE2 was an independent risk factor for major adverse cardiac events in patients with aortic stenosis [4] and coronary artery disease [5]. Others have recently shown that higher plasma ACE2

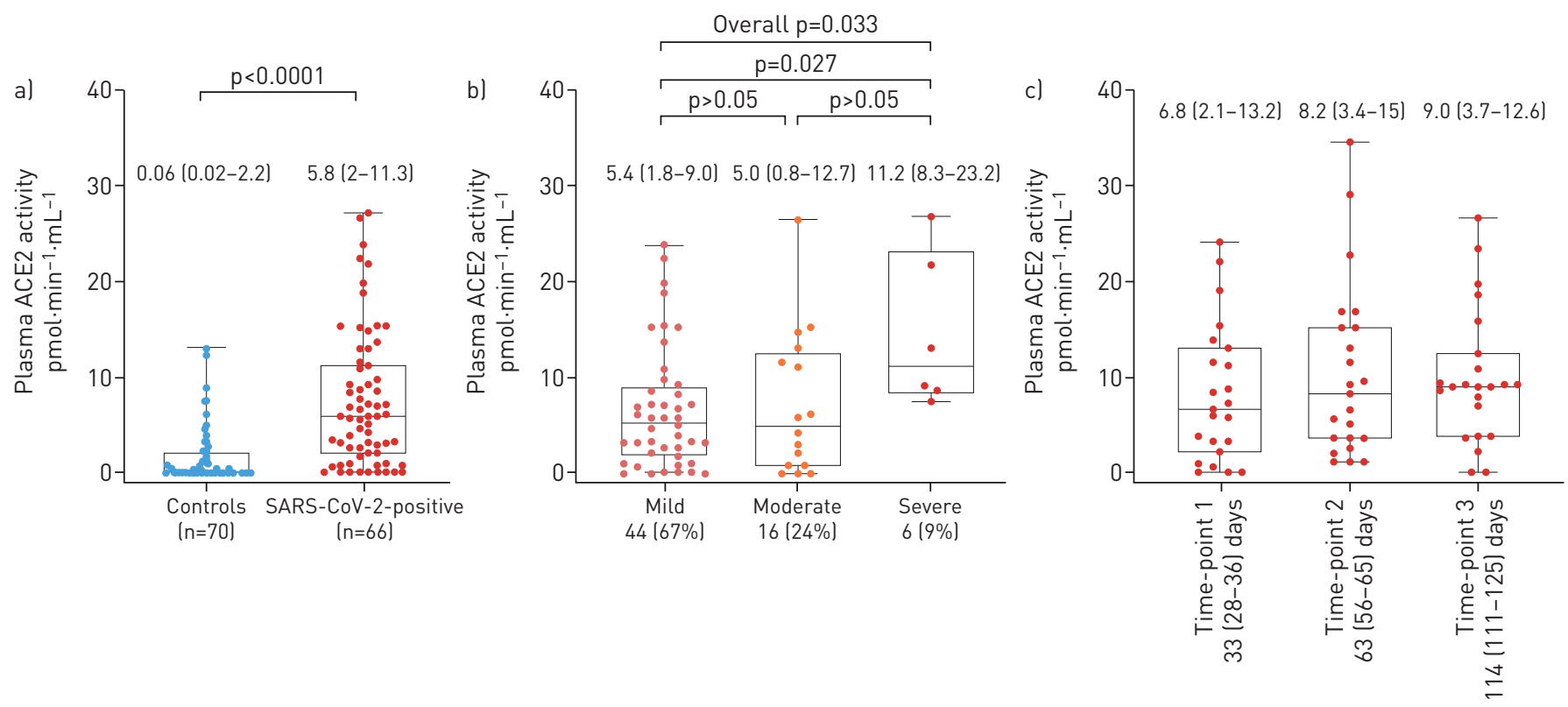

FIGURE 1 Plasma angiotensin converting enzyme 2 (ACE2) activity is elevated in patients who recovered from severe acute respiratory syndromecoronavirus-2 (SARS-CoV-2) infection (a), is increased with disease severity (b), and remains elevated 2-3 months after SARS-CoV-2 infection. Data shown as scatter points and box plot with whiskers representing minimum and maximum values of median plasma ACE2 activity between controls, and patients who recovered from SARS-CoV-2 infection (a) and median plasma ACE2 activity in patients recovered from SARS-CoV-2 infection according to disease severity (b). The median (interquartile range) plasma ACE2 activity levels are shown above each bar. The median plasma ACE2 activity in patients recovered from SARS-CoV-2 infection with serial samples ( $n=23$ ) at three time-points (indicated by median (interquartile range) days after positive PCR test) are shown in (c). The median (interquartile range) plasma ACE2 activity levels are shown above the bar for each time-point. Two-tailed $p$-values $<0.05$ were considered significant. 
concentration was associated with increased risk of major cardiovascular events in a large global population-based study [8].

In conclusion, we suggest that binding of SARS-CoV-2 to membrane ACE2 has at least two effects. First, it downregulates membrane ACE2 causing a dysregulated local renin angiotensin system (RAS) that favours inflammation and ongoing tissue damage secondary to excess angiotensin II [9]. Second, the dysregulated local RAS is associated with prolonged shedding of the catalytically active site of ACE2 into the circulation.

In addition to the early multisystem effects of COVID-19, a proportion of patients experience prolonged ill-health commonly known as "long-COVID", the nature of which remains to be fully characterised [10]. Larger studies are now needed to determine if persistently elevated plasma ACE2 activity may identify people at risk of prolonged illness or cardiac events following COVID-19 infection, as well as studies that address whether therapeutic strategies directed at a dysregulated RAS can reduce COVID-19 complications.

Sheila K. Patel ${ }^{1,4}$, Jennifer A. Juno ${ }^{2,4}$, Wen Shi Lee ${ }^{2}$, Kathleen M. Wragg ${ }^{2}$, P. Mark Hogarth ${ }^{3}$, Stephen J. Kent ${ }^{2,5}$ and Louise M. Burrell $\oplus^{1,5}$

${ }^{1}$ Dept of Medicine, Austin Health, University of Melbourne, Melbourne, Australia. ${ }^{2}$ Dept of Microbiology and Immunology, University of Melbourne at the Peter Doherty Institute for Infection and Immunity, Melbourne, Australia. ${ }^{3}$ Immune Therapies Group, Burnet Institute, Melbourne, Australia. ${ }^{4}$ Contributed equally. ${ }^{5}$ S.J. Kent and L.M. Burrell contributed equally to this article as lead authors and supervised the work.

Correspondence: Louise M. Burrell, Dept of Medicine, University of Melbourne, Lance Townsend Building, Austin Health, Studley Road, Melbourne, Victoria 3084, Australia. E-mail: 1.burrell@unimelb.edu.au

Received: 6 Oct 2020 | Accepted: 3 Jan 2021

Acknowledgements: We thank the generous participation of the study subjects for providing samples. We thank Adam Wheatley, Jane Batten and Helen Kent for help with the COVID-19 cohort, and Ping Huang and Thomas McConville for assistance with the ACE2 activity assays.

Conflict of interest: S.K. Patel has nothing to disclose. J.A. Juno has nothing to disclose. W.S. Lee has nothing to disclose. K.M. Wragg has nothing to disclose. P.M. Hogarth reports grants from National Health and Medical Research Council (Australia) during the conduct of the study; grants from Genmab (b.v.), outside the submitted work. S.J. Kent has nothing to disclose. L.M. Burrell has nothing to disclose.

Support statement: This study was supported by the Victorian Government (S.J. Kent, J.A. Juno), an Australian government Medical Research Future Fund award GNT2002073 (S.J. Kent, P.M. Hogarth), the ARC Centre of Excellence in Convergent Bio-Nano Science and Technology CE140100036 (S.J. Kent), NHMRC programme grants APP1149990 (S.J. Kent) and APP 1055214 (L.M. Burrell), project grant GNT1145303 (P.M. Hogarth), Medical Research Future Fund award GNT 1175865 (S.K. Patel, L.M. Burrell), Austin Medical Research Foundation Grant (S.K. Patel, L. M. Burrell). J.A. Juno and S.J. Kent are supported by NHMRC fellowships. Funding information for this article has been deposited with the Crossref Funder Registry.

\section{References}

1 Huang C, Wang Y, Li X, et al. Clinical features of patients infected with 2019 novel coronavirus in Wuhan, China. Lancet 2020; 395: 497-506.

2 Hoffmann M, Kleine-Weber H, Schroeder S, et al. SARS-CoV-2 cell entry depends on ACE2 and TMPRSS2 and is blocked by a clinically proven protease inhibitor. Cell 2020; 181: 271-280.

3 Lew RA, Warner FJ, Hanchapola I, et al. Angiotensin-converting enzyme 2 catalytic activity in human plasma is masked by an endogenous inhibitor. Exp Physiol 2008; 93: 685-693.

4 Ramchand J, Patel SK, Kearney LG, et al. Plasma ACE2 activity predicts mortality in aortic stenosis and is associated with severe myocardial fibrosis. JACC Cardiovasc Imaging 2020; 13: 655-664.

5 Ramchand J, Patel SK, Srivastava PM, et al. Elevated plasma angiotensin converting enzyme 2 activity is an independent predictor of major adverse cardiac events in patients with obstructive coronary artery disease. PLoS One 2018; 13: e0198144.

6 COVID-19 Treatment Guidelines Panel. Coronavirus Disease 2019 (COVID-19) Treatment Guidelines. National Institutes of Health. www.covid19treatmentguidelines.nih.gov/ Date last accessed: 23 December 2020.

7 Juno JA, Tan HX, Lee WS, et al. Humoral and circulating follicular helper T cell responses in recovered patients with COVID-19. Nat Med 2020; 26: 1428-1434.

8 Narula S, Yusuf S, Chong M, et al. Plasma ACE2 and risk of death or cardiometabolic diseases: a case-cohort analysis. Lancet 2020; 396: 968-997.

9 Ramchand J, Burrell LM. Circulating ACE2: a novel biomarker of cardiovascular risk. Lancet 2020; 396: 937-939.

10 Yelin D, Wirtheim E, Vetter P, et al. Long-term consequences of COVID-19: research needs. Lancet Infect Dis 2020; 20: 1115-1117. 